\title{
Microglial responses to CSF1 overexpression do not promote the expansion of other glial lineages
}

\author{
Ishani De ${ }^{1 \dagger}$, Vilena Maklakova ${ }^{2 \dagger}$, Suzanne Litscher ${ }^{2 \dagger}$, Michelle M. Boyd ${ }^{2}$, Lucas C. Klemm', Ziyue Wang ${ }^{3}$, \\ Christina Kendziorski ${ }^{4,5}$ and Lara S. Collier ${ }^{1,2,5^{*}}$ (i)
}

\begin{abstract}
Background: Colony-stimulating factor 1 (CSF1) expression in the central nervous system (CNS) increases in response to a variety of stimuli, and CSF1 is overexpressed in many CNS diseases. In young adult mice, we previously showed that CSF1 overexpression in the CNS caused the proliferation of $\mid B A 1^{+}$microglia without promoting the expression of M2 polarization markers.

Methods: Immunohistochemical and molecular analyses were performed to further examine the impact of CSF1 overexpression on glia in both young and aged mice.

Results: As CSF1 overexpressing mice age, IBA $1^{+}$cell numbers are constrained by a decline in proliferation rate. Compared to controls, there were no differences in expression of the M2 markers ARG1 and MRC1 (CD206) in CSF1 overexpressing mice of any age, indicating that even prolonged exposure to increased CSF1 does not impact M2 polarization status in vivo. Moreover, RNA-sequencing confirmed the lack of increased expression of markers of M2 polarization in microglia exposed to CSF1 overexpression but did reveal changes in expression of other immunerelated genes. Although treatment with inhibitors of the CSF1 receptor, CSF1R, has been shown to impact other glia, no increased expression of oligodendrocyte lineage or astrocyte markers was observed in CSF1 overexpressing mice.

Conclusions: Our study indicates that microglia are the primary glial lineage impacted by CSF1 overexpression in the CNS and that microglia ultimately adapt to the presence of the CSF1 mitogenic signal.
\end{abstract}

Keywords: CSF1, Microglia, Astrogliosis, Oligodendrogenesis

\section{Introduction}

CNS resident macrophages (microglia) are known to have important roles in both CNS homeostasis and disease. Macrophage lineage cells including microglia express CSF1R, a receptor tyrosine kinase, which is activated by its

\footnotetext{
* Correspondence: Icollier@wisc.edu

${ }^{\dagger}$ Ishani De, Vilena Maklakova, and Suzanne Litscher made equal contributions.

${ }^{1}$ Molecular and Cellular Pharmacology Graduate Program, University of Wisconsin, Madison, USA

${ }^{2}$ Pharmaceutical Sciences Division, School of Pharmacy, University of Wisconsin, Madison, USA

Full list of author information is available at the end of the article
}

ligands CSF1 and Interleukin-34 (IL-34). Mice genetically deficient for CSF1R have severe reductions in several macrophage populations including microglia [1]. In the normal brain, CSF1 and IL-34 have different expression patterns and therefore have regional-specific impacts on microglia [2-5]. During mouse neonatal development, whole-brain expression of Csf1r and its ligands peak during the 2nd and 3rd postnatal weeks, respectively, before declining [6]. This corresponds with the time that microglial numbers undergo rapid developmental changes. Specifically, microglial numbers peak during the 2nd postnatal week and then decline during the 3rd postnatal

C C The Author(s). 2021 Open Access This article is licensed under a Creative Commons Attribution 4.0 International License, which permits use, sharing, adaptation, distribution and reproduction in any medium or format, as long as you give appropriate credit to the original author(s) and the source, provide a link to the Creative Commons licence, and indicate if changes were made. The images or other third party material in this article are included in the article's Creative Commons licence, unless indicated otherwise in a credit line to the material. If material is not included in the article's Creative Commons licence and your intended use is not permitted by statutory regulation or exceeds the permitted use, you will need to obtain permission directly from the copyright holder. To view a copy of this licence, visit http://creativecommons.org/licenses/by/4.0/ The Creative Commons Public Domain Dedication waiver (http://creativecommons.org/publicdomain/zero/1.0/) applies to the data made available in this article, unless otherwise stated in a credit line to the data. 
week due to both decreased proliferation and increased apoptosis [6]. In the adult, microglia do continue to proliferate at a low rate, but it has been observed that this proliferation is balanced by a similar rate of apoptosis [7]. Studies in mouse models utilizing CSF1R inhibitors indicate that the CSF1R signaling axis is important for both microglial proliferation and survival in the normal adult [7-9]. In situations of disease or injury, Csf1 expression is often upregulated, which can influence microglial homeostasis [10-12].

Activated macrophages, including microglia, can be classified as being polarized to an M1 (pro-inflammatory) or M2 (immunosuppressive) phenotype [13]. In in vitro macrophage cultures, CSF1 has been proposed to promote M2-like phenotypes [14-16]. In the context of a high-grade brain tumor model where gliomaassociated macrophages/microglia (GAMs) were M2 polarized, CSF1R inhibitors were found to decrease expression of M2 markers in GAMs such as Arg1 and Mrc1 (CD206) without influencing their numbers [17, 18]. However, the M1/M2 classification is highly simplified, and a wide variety of activation states for macrophages have been found $[19,20]$.

In normal adult mice, CSF1R expression is reported to be confined to microglia and some neurons [3, 12], yet treatment with CSF1R inhibitors can impact other glia. For example, increases in expression of astrocytic markers such as Gfap were observed upon treatment with a CSF1R inhibitor in some, but not all, studies $[8,21]$. Decreased numbers of oligodendrocyte lineage cells were observed in certain brain regions in both Csf1r deficient mice and mice treated with certain CSF1R inhibitors $[9,22]$. However, CSF1R inhibitor-mediated microglial depletion can be achieved without impacting oligodendrocyte lineage cells, suggesting potential off-target effects of these inhibitors [22]. Nevertheless, microglia have been shown to produce factors that influence oligodendrocyte precursor cell (OPC) proliferation, survival, or differentiation [23, 24]; however, it is not known if increasing microglial numbers is sufficient to impact oligodendrocyte lineage cells.

To study the role of increased CSF1 expression in the $\mathrm{CNS}$, we previously generated transgenic mice that overexpress the secreted form of CSF1 in a subset of GFAP ${ }^{+}$ cells utilizing the TRE/tTA system (hereafter referred to as CSF1 OE mice). Previously, we examined the response of $\mathrm{IBA}^{+}$microglia to CSF1 OE in young adult mice [21]. Here, we expand upon those studies to examine responses to CSF1 OE in both microglia and other glia in young and aged mice.

\section{Materials and methods}

\section{Mice}

Mouse experiments were performed according to the institutional guidelines for animal care under the approval of the Institutional Animal Care and Use Committee of the University of Wisconsin, Madison. CSF1 OE mice have been described previously [21]. The genetic backgrounds of mice used for this study were F1s of CD1 to C57Bl/6 (immunofluorescence and CD11b ${ }^{+}$cell enrichment) or $\mathrm{C} 57 \mathrm{Bl} / 6$ (RNA isolation from half brain hemispheres).

\section{Fluorescence and immunofluorescence}

For EGFP imaging, isolated brains were fixed in 4\% PFA, sunk through sucrose, and embedded in OCT for frozen sectioning. Sections were washed in PBS before staining with DAPI for imaging. For immunofluorescence, slides from formalin-fixed, paraffin-embedded brains were rehydrated to water through a graded alcohol series and antigen retrieval performed in $\mathrm{pH} 6$ citrate buffer (Vector laboratories) with $0.02 \%$ TWEEN-20 added, following previously described procedures [21]. Antibodies and dilutions are described in Table 1. When needed, DyLight 649 labeled Lycopersicon Esculentum Lectin (DL-1178, Vector laboratories) was used at 1:300 before antibody staining. The Deadend TUNEL kit (Promega) was used to label apoptotic cells as previously described [21]. For cell counting in Image $[25,26]$, images of $\mathrm{z}$ stacks of 10 steps $1 \mu$ apart were used. For cell counting for each cell type, total cell numbers were determined by counting nuclei (DAPI). For cytoplasmic (IBA1 and GFAP) and cell surface (PDGF RA) antigens, a cell was considered positive if the signal surrounded the nucleus. For IBA1, GFAP, and OLIG2, PDGFA cell counts data are presented as the percent of total cells positive for the marker of interest (e.g., number of $\mathrm{IBA}^{+}$cells divided by the total number of cells times $100 \%)$. For IBA1 cell counts in the midbrain and brainstem, $1060 \times$ fields were counted per brain region per mouse. For OLIG2, PDGFRA, or GFAP cell counting, a minimum of 1200 cells in the cerebellar white matter or cortex were counted. For IBA $1^{+}$cell proliferation, a minimum of $100 \mathrm{IBA}^{+}$cells per brain region were examined per mouse. For microglial apoptosis, a minimum of 200 $\mathrm{IBA}^{+}$cells per brain region were examined per mouse.

\section{Statistics}

With the exception of RNA-seq analysis, Prism (GraphPad) was used to perform statistical analyses and to produce graphs. All data were analyzed by unpaired, twotailed $t$ test with the exception of brainstem 6-month apoptotic cells which were analyzed by Wilcoxon signed-rank test. In all figures, error bars indicate standard deviation. Unless otherwise indicated, $n=3$ to 4 mice per group.

\section{RNA isolation from half brain hemispheres}

Tissue was homogenized in TRIzol and purified using the TRIzol Plus RNA Purification Kit (Thermo Fisher) 
Table 1 Antibodies and dilutions and utilized in this study

\begin{tabular}{|c|c|c|c|c|c|c|}
\hline \multicolumn{7}{|c|}{ Primary antibodies } \\
\hline Target & Host species & Catalog \# & Manufacturer & Dilution & RRID & \\
\hline $\mid \mathrm{BA} 1$ & Rabbit & 019-19741 & Wako & $1: 200$ & AB_839504 & \\
\hline IBA1 & Goat & ab48004 & Abcam & $1: 150$ & AB_870576 & \\
\hline ARG1 & Rabbit & ab91279 & Abcam & $1: 200$ & AB_10674215 & \\
\hline MRC1 (CD206) & Rabbit & ab64693 & Abcam & $1: 1000$ & AB_1523910 & \\
\hline Ki67 & Mouse & 550609 & BD Biosciences & $1: 200$ & AB_393778 & \\
\hline OLIG2 & Rabbit & AB9610 & Millipore Sigma & $1: 150$ & AB_570666 & \\
\hline GFAP & Chicken & $a b 4674$ & Abcam & $1: 200$ & AB_304558 & \\
\hline GFAP & Rabbit & ab7260 & Abcam & $1: 100$ & AB_305808 & \\
\hline CSF1 & Goat & AF416 & R\&D systems & $1: 25$ & AB_355351 & \\
\hline PDGFRA & Goat & AF1062 & R\&D systems & $1: 200$ & AB_2236897 & \\
\hline \multicolumn{7}{|c|}{ Secondary antibodies } \\
\hline Target & Host species & Catalog \# & Manufacturer & Dilution & RRID & Conjugation \\
\hline Anti-mouse & Goat & ab97239 & Abcam & $1: 200$ & AB_10680851 & FITC \\
\hline Anti-rabbit & Donkey & ab150076 & Abcam & $1: 200$ & AB_2782993 & Alexa 594 \\
\hline Anti-chicken & Goat & ab150175 & Abcam & $1: 200$ & AB_2732800 & Alexa 647 \\
\hline Anti-goat & Donkey & ab150129 & Abcam & $1: 250$ & AB_2687506 & Alexa 488 \\
\hline Anti-goat & Donkey & A21447 & Invitrogen & $1: 200$ & AB_141884 & Alexa 647 \\
\hline Anti-mouse & Horse & MKB-2225 & Vector Laboratories & $1: 250$ & AB_2336564 & biotin $^{a}$ \\
\hline
\end{tabular}

${ }^{\mathrm{a}}$ Followed by $1 \mathrm{~h}$ incubation in Streptavidin-FITC (eBioscience, 11-4317-87) at 1:100 dilution

RRID Research Resource Identifier

including an on-column DNAse digestion. Postisolation, the TURBO DNA free kit (Thermo Fisher) was used to eliminate any residual contaminating genomic DNA before further analysis.

\section{Reverse transcription, qualitative PCR (RT-qPCR)}

cDNA was generated with the High-Capacity cDNA Reverse Transcription Kit (Thermo Fisher). Real-time PCR was completed using Step One Plus Real-Time PCR System and Power UP SYBR green (Applied Biosystems). Gene expression was normalized to $T b p$ and $2^{-\Delta C t}$ values were calculated. Primers sequences are provided in Table 2. Data in figures are presented as relative expression levels compared to control mice which are normalized to one.

\section{Microglia enrichment}

CSF1 OE or control mice (ages p14 or p15, $n=4$ per group) were perfused with PBS following a fatal dose of pentobarbital sodium. Brains were isolated and bisected sagittally. Left brain hemispheres were formalin-fixed for other studies and microglia were enriched from the right

Table 2 Primers utilized in this study

\begin{tabular}{|c|c|c|}
\hline Gene & Forward primer 5'-3' & Reverse primer 5'-3' \\
\hline $\operatorname{Arg} 1$ & AGACATCGTGTACATTGGCTTGCG & CCCAGCTTGTCTACTTCAGTCATGGA \\
\hline C3 & ACAAGAACACCCTCATCATCTAC & GGCTGGATAAGTCCCACATT \\
\hline Csf1 & GGCATCATCCTAGTCTTGCTG & ACCTGTCTGTCCTCATCCT \\
\hline Gfap & ACATGCAAGAGACAGAGGAGTGGT & AGTCGTTAGCTTCGTGCTTGGCTT \\
\hline Mog & GCTTCTTCAGAGACCACTCTT & GATAGGCACAAGTGCGATGA \\
\hline $\mathrm{MrCl}^{*}$ & TATCTCTGTCATCCCTGTCTCT & CAAGTTGCCGTCTGAACTGA \\
\hline Olig2 & AGCGAGCACCTCAATCTAAT & GGGATGATCTAAGCTCTCGAA \\
\hline Pdgfr alpha & GACGAGACCATCGAGGACAT & GCCTCGGGAACTTTCTCTCT \\
\hline $\operatorname{SIcla2} *$ & AAAGAATCGCCCACCACAT & CCATGCTCCTCATTCTCACAG \\
\hline$T b p$ * & TTCACCAATGACTCCTATGACC & CAAGTTTACAGCCAAGATTCACG \\
\hline
\end{tabular}

* indicates primers ordered pre-designed from IDT 
hemisphere by pull-down utilizing CD11b-conjugated magnetic beads (Miltenyi Biotech) using published methods with the Percoll (GE Healthcare) method for myelin removal [5]. Cell pellets were suspended in TRIzol (Thermo Fisher) and RNA was purified using the TRIzol Plus Purification kit including an on-column DNAse digestion step (Thermo Fisher).

\section{RNA sequencing (RNA-seq)}

RNA quality and quantity were assayed with the RNA 6000 Pico Kit (Agilent) and Quant-iT RiboGreen RNA Assay Kit (Thermo Fisher). Libraries for RNA-seq were generated using the TruSeq RNA Library Prep Kit v2 (Illumina). 2X125 reads were obtained from one lane of the HiSeq 2500 system (Illumina).

\section{RNA-seq data analysis}

Reads were mapped back to the genome using the short read aligner Bowtie v1.0.0 [6], followed by RSEM v1.2.7 [7] to estimate gene expression. Analyses were carried out in $\mathrm{R}$ [8], a publicly available statistical analysis environment. Specific software packages were obtained from Bioconductor [9] unless otherwise noted. EBSeq v1.14.0 [10] was used with default parameters to calculate the posterior probability of a gene being differentially expressed (DE). A gene was identified as being DE if its posterior probability exceeded 0.95 (which controls the overall False Discovery Rate (FDR) at 5\%) and the posterior fold change (estimated from the empirical Bayes model) was less than 0.7 (or greater than $1.43(1 / 0.7)$ ).

Functional annotations were performed using the Database for Annotation, Visualization and Integrated Discovery (DAVID) [27, 28], and data presented are terms with Benjamini-Hochberg corrected $p$ values < 0.05 to control the FDR at $5 \%$.

\section{Results}

Proliferation rates of $\mathrm{IBA} 1^{+}$cells decline over time in CSF1 OE mice

Previously, an increased rate of $\mathrm{IBA}^{+}$microglial proliferation and increased $\mathrm{IBA}^{+}$microglial numbers were observed in young adult CSF1 OE mice compared to controls [21]. Continued expansion of $\mathrm{IBA}^{+}$cells could have detrimental effects in the CNS; therefore, it was hypothesized that responses to CSF1 overexpression would need to change over time. To examine if adaptation to increased CSF1 levels occurs over time, $\mathrm{IBA}^{+}$cell counts and proliferation rates were examined in young and aged CSF1 OE and control mice (representative images in Supplemental Figure 1). To be consistent with a past study of CSF1 OE mice, IBA1 counts were performed in the brain stem and midbrain, two regions known to harbor high levels of CSF1 transgene expression [21]. IBA1 counts confirmed that $\mathrm{IBA}^{+}$cell numbers were increased in CSF1 OE mice compared to controls at all ages examined (Fig. 1A). Next, Ki67 staining was used to identify proliferating $\mathrm{IBA}^{+}$cells. At both p14 and 6 months, microglial proliferation rates were higher in CSF1 OE mice compared to controls, but by 1 year of age, microglial proliferation rates were equivalent (Fig. 1B). This difference in aged mice is not due to transgene silencing as RT-qPCR detects approximately 2.5-fold increased Csf1 expression in p14 CSF1 OE mice compared to control, and an approximately 3fold increase in Csf1 expression in 1-year-old CSF1 OE mice compared to 1-year-old control mice (Supplemental Figure 2A and 2B). Additionally, EGFP (encoded as part of the TRE-CSF1 transgene) and CSF1 proteins are also readily detected in 1-year-old CSF1 OE mice (Supplemental Figure 2C and 2D). TUNEL analysis indicates that CSF1 OE does not influence apoptosis rates (Fig. 1C; representative images in Supplemental Figure 3), indicating that downregulation of the proliferative response likely constrains $\mathrm{IBA}^{+}$cell expansion in CSF1 OE mice.

\section{CSF1 OE does not impact the expression of the M2} polarization markers ARG1 and MRC1, even in aged mice Although CSF1 has been proposed to be a factor that can polarize macrophages toward an M2 phenotype [14-16], increased expression of markers of M2 polarization was not previously observed in young adult CSF1 OE mice [21]. To determine if continued exposure to CSF1 OE promotes an M2 phenotype, immunofluorescence staining was performed for two commonly used M2 markers, ARG1 and MRC1 (CD206) [13], in young and aged mice. ARG1 was not detectable in IBA1 ${ }^{+}$cells in either p14 or 1-year OE mice (Fig. 2A; validation of ARG1 antibody efficacy can be found in Supplemental Figure 4). MRC1 expression as detected by immunofluorescence was observed, as expected, in perivascular macrophages but was not observed in parenchymal $\mathrm{IBA}^{+}$cells in either p14 or 1-year OE mice (Fig. 2B). RT-qPCR also did not detect a difference in Arg1 (Fig. 2C) or Mrc1 (Fig. 2D) expression between the brains of control and CSF1 OE mice at either p14 or 1 year. Taken together, this data provides additional evidence that CSF1 as the only stimulus does not impact microglial polarization toward an M2 phenotype in vivo.

\section{CSF1 OE impacts the expression of genes involved in translation and the immune response in microglia}

To further examine the impact of CSF1 OE on microglia, CD11b-conjugated magnetic beads were used to enrich for microglia in CSF1 OE or control mice for RNA-seq. Three hundred fourteen genes were found to be differentially expressed between control and CSF1 OE (Fig. 3A and Supplemental Table 1). Hierarchical clustering found 
a
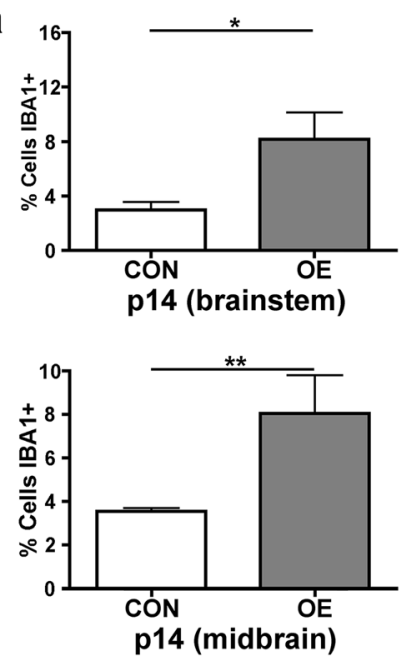

b
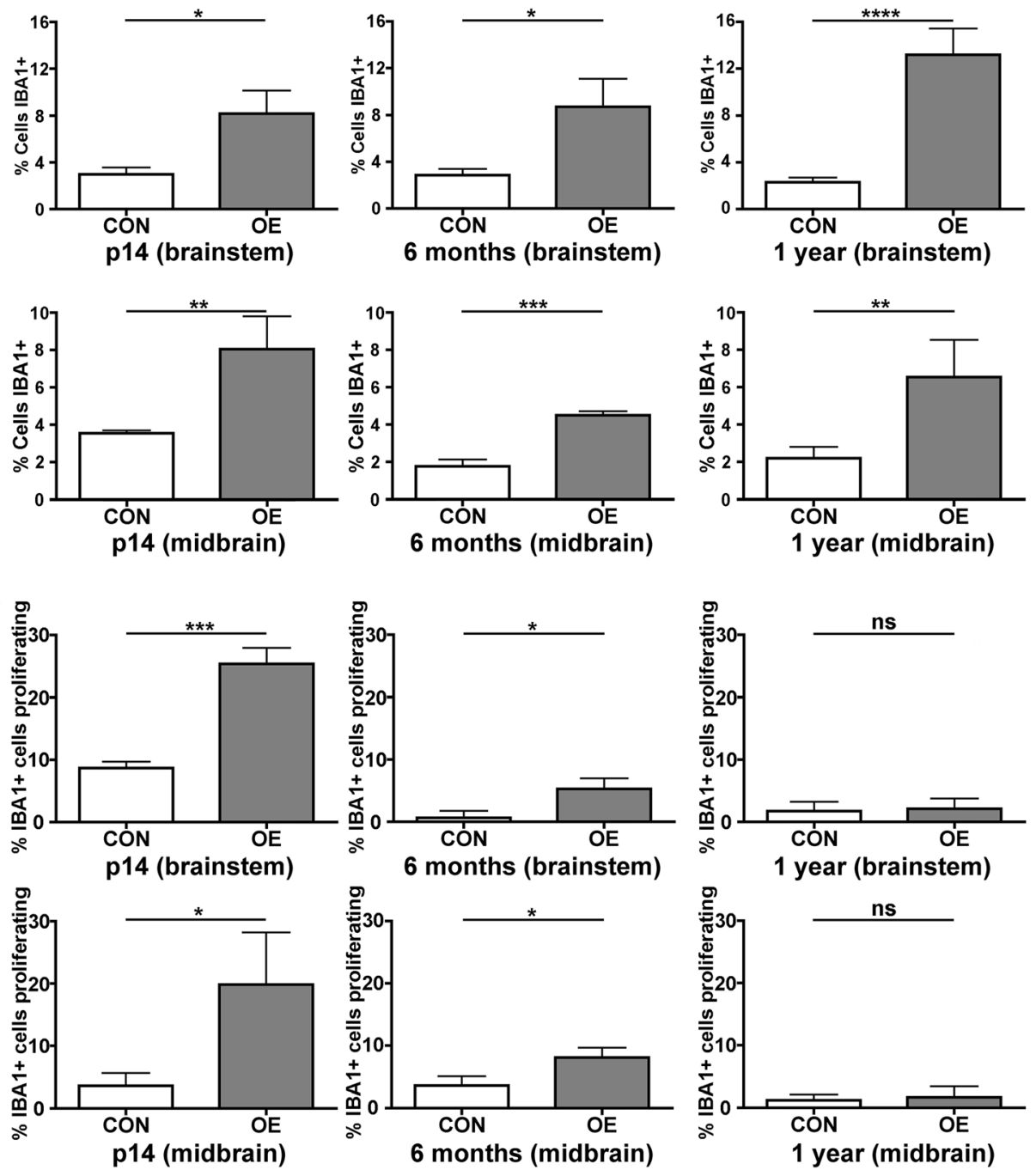

\section{C}
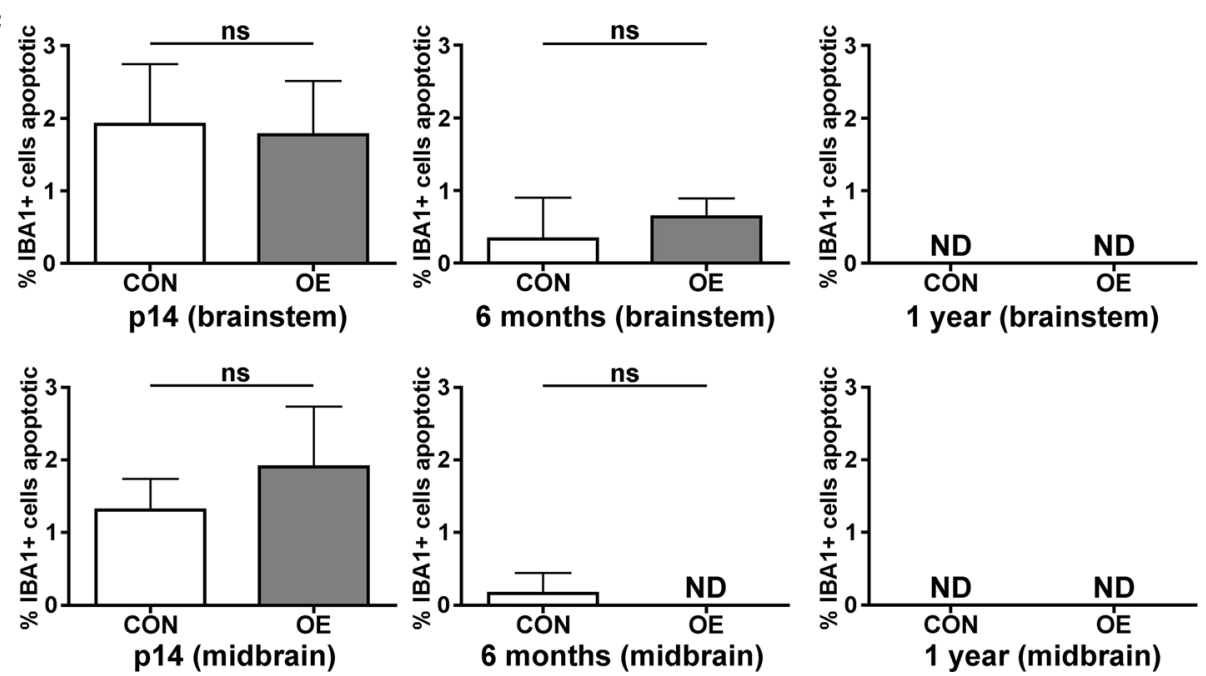

Fig. 1 (See legend on next page.) 
(See figure on previous page.)

Fig. $1 \mathrm{~A}$ decline in proliferation rate constrains $\mid \mathrm{BA} \mathrm{I}^{+}$cell numbers as CSF1 OE mice age. Cells were quantified in both the brainstem and midbrain for both control (CON, white bars) and CSF1 OE (OE, grey bars) mice. A Quantification of the percent of cells that are IBA $1^{+}$. B Quantification of the percent of $|\mathrm{BA}|^{+}$cells that are proliferating $\left(\mathrm{Ki} 67^{+}\right)$. C Quantification of the percent of $\mid \mathrm{BA} 1^{+}$cells that are apoptotic $\left(\mathrm{TUNEL}{ }^{+} \mid \mathrm{BA} 1^{+}\right)$. ND $=n \mathrm{none}$ detected. ns = non-significant $(p<0.05) ;{ }^{*} p<0.05 ;{ }^{* *} p<0.01 ;{ }^{* * *} p<0.001$; and ${ }^{* * * *} p<0.0001$ by unpaired, two-tailed $t$ test with the exception of brainstem 6-month apoptotic cells which was analyzed by Wilcoxon signed-rank test

that samples of the same genotype grouped together. No commonly used markers for M2 phenotypes $[29,30]$ had increased expression in CSF1 OE microglia, and two (Ccl24 and Retnla (Fizz1)) had decreased expression compared to control. To determine which cellular processes are impacted when microglia are exposed to CSF1 overexpression, functional annotation was performed using DAVID (Fig. 3B). Most GO biological process (bp) terms enriched in differentially expressed genes were related to either translation or immune system activities. Transcripts encoding multiple cytoplasmic ribosomal proteins were upregulated in CSF1 OE while several additional subunits had increased expression in CSF1 OE microglia but did not meet the threshold to be considered differentially expressed (data not shown). Other genes involved in ribosome biogenesis or translation, including Eef1b2, Rbm3, $N h p 2$, and Npm1, were also upregulated in CSF1 OE microglia. Multiple genes involved in different aspects of immune responses were also differentially expressed, including both transcripts encoding secreted molecules like

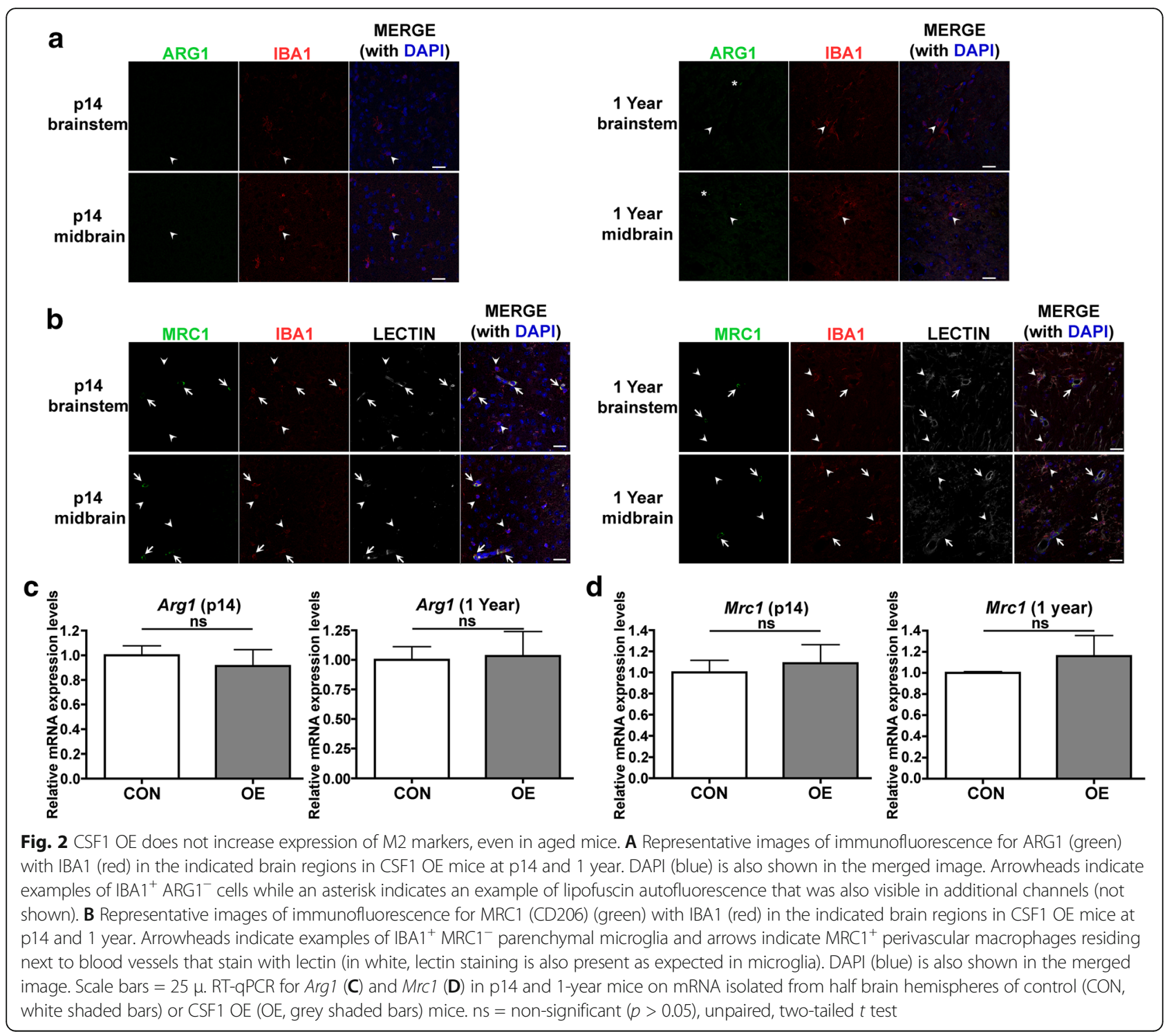


a

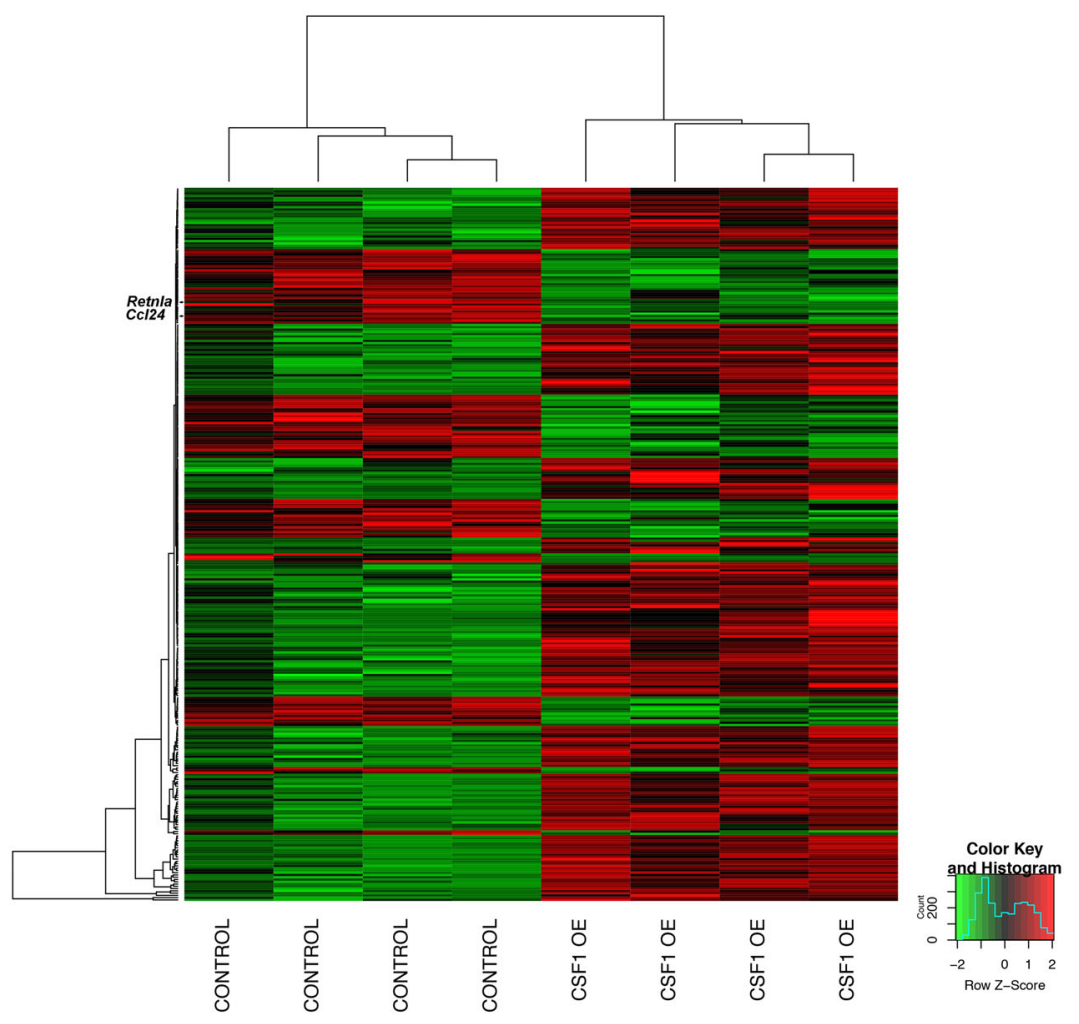

b

\begin{tabular}{|l|c|c|}
\hline \multicolumn{1}{|c|}{ GO bp term } & Fold Enrichment & p value \\
\hline $\begin{array}{l}\text { antigen processing and presentation of exogenous } \\
\text { peptide antigen via MHC class I, TAP-dependent }\end{array}$ & 15.98 & 0.001 \\
$\begin{array}{l}\text { antigen processing and presentation of peptide antigen } \\
\text { via MHC class I }\end{array}$ & 13.32 & 0.003 \\
\hline $\begin{array}{l}\text { cellular response to interferon-gamma } \\
\text { defense response to virus }\end{array}$ & 7.05 & 0.049 \\
\hline $\begin{array}{l}\text { immune system process } \\
\text { inflammatory response }\end{array}$ & 5.74 & $3.17 \mathrm{E}-04$ \\
\hline $\begin{array}{l}\text { innate immune response } \\
\text { negative regulation of neuron projection development }\end{array}$ & 6.62 & $5.34 \mathrm{E}-16$ \\
\hline $\begin{array}{l}\text { positive regulation of T cell mediated cytotoxicity } \\
\text { positive regulation of T cell proliferation }\end{array}$ & 3.38 & 0.008 \\
\hline response to lipopolysaccharide & 7.49 & $3.92 \mathrm{E}-05$ \\
\hline response to virus & 18.02 & 0.038 \\
\hline ribosomal small subunit assembly & 8.56 & 0.022 \\
translation & 4.17 & 0.007 \\
\hline
\end{tabular}

Fig. 3 A Heatmap of 314 differentially expressed genes for control versus CSF1 OE samples. Each row represents a single gene, while each column represents a sample. Two hundred fourteen genes are upregulated (shown in red) in the CSF1 OE condition and one hundred are downregulated (shown in green). Genes are shown in the order depicted in Supplemental Table 1, and the rows for the M2 markers Cc124 and Retnla are indicated. B GO terms enriched in differentially expressed genes between microglia from CSF1 OE and control mice. Benjamini-Hochberg corrected $p$ values are presented

$\mathrm{Cfb}$ and cell surface receptors like TLR2. MHC class I genes and additional interferon-regulated genes such as Oas family members and Usp 18 were also upregulated in microglia from CSF1 OE mice. In summary, RNA-seq data support the lack of increased expression of M2 polarization markers in microglia exposed to CSF1 OE; and also indicate that CSF1 OE exposed microglia do have some phenotypic differences from control microglia.
CSF1 OE does not increase the expression of oligodendrocyte lineage markers

There are several lines of evidence indicating that normal microglia influence oligodendrogenesis [23, 24]. To determine if CSF1 overexpression impacts oligodendrocyte lineage cells, $\mathrm{OLIG}^{+}$; $\mathrm{PDGFRA}^{+}$oligodendrocyte precursor cells (OPCs) as well as maturing or mature oligodendrocyte lineage $\left(\mathrm{OLIG}^{+}\right.$; $\left.\mathrm{PDGFRA}^{-}\right)$cells were 
a

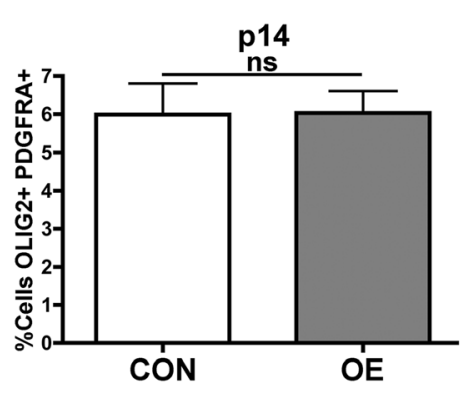

b

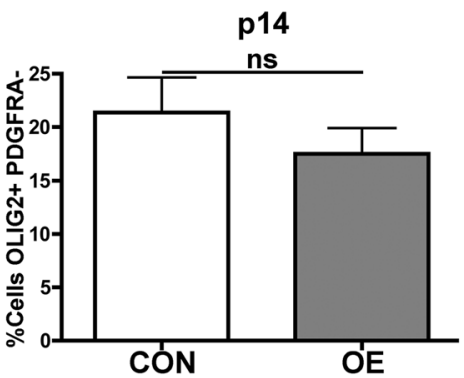

C

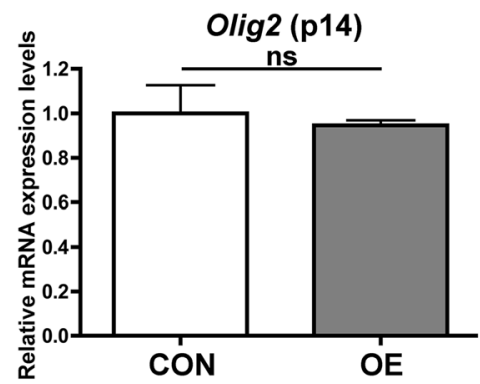

d

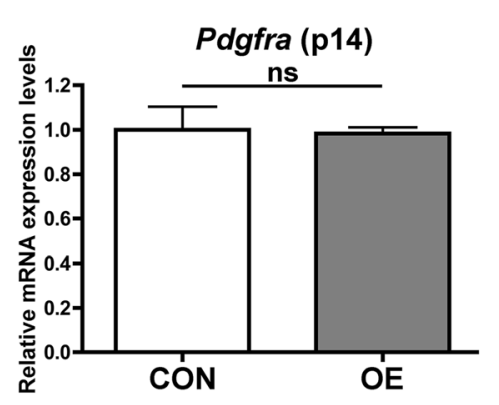

e

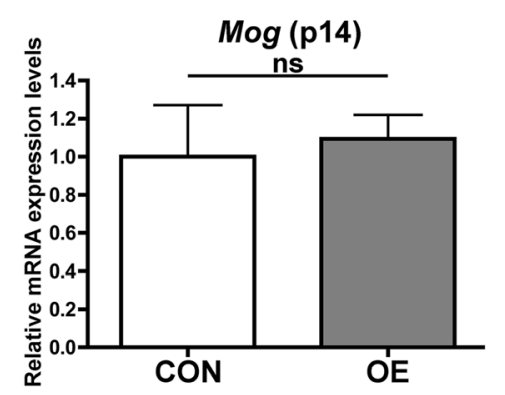

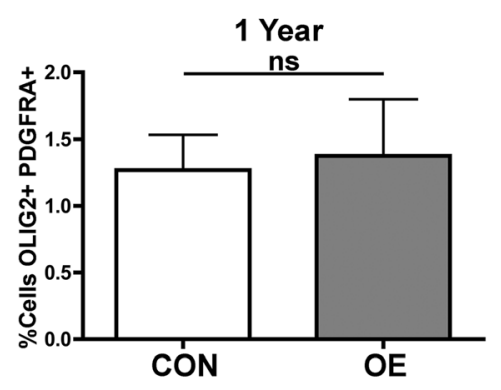
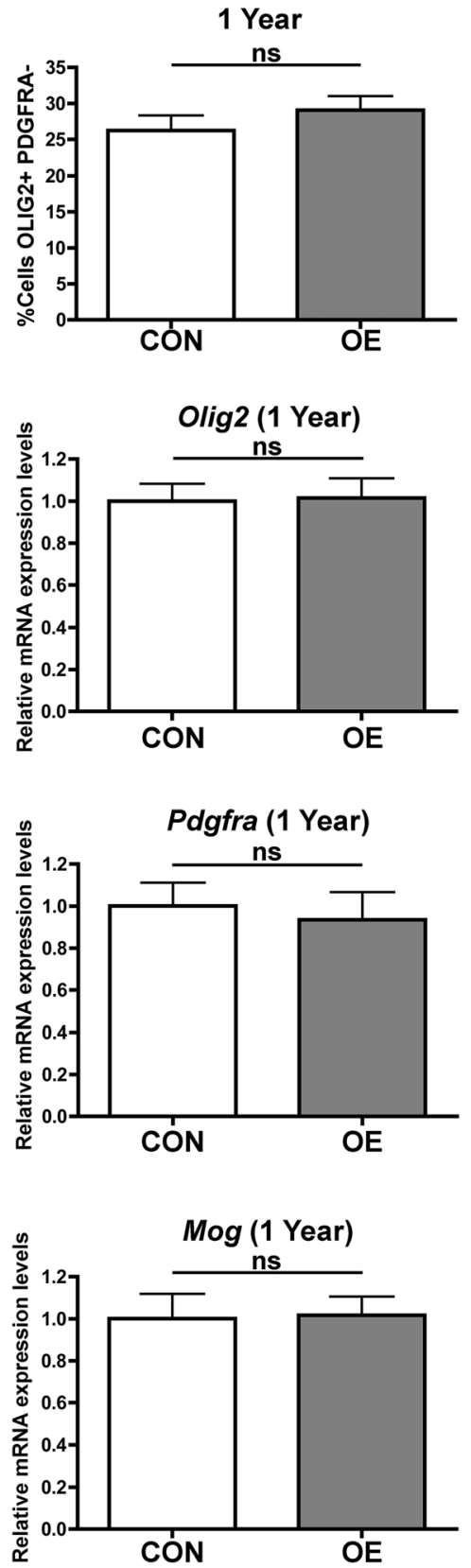

Fig. 4 (See legend on next page.) 
(See figure on previous page.)

Fig. 4 CSF1 OE does not impact the expression of genes expressed in the oligodendrocyte lineage. Quantification of the percent of cells that are OPCS (OLIG2 ${ }^{+}$; PDGFRA $\left.{ }^{+}\right)(\mathbf{A})$ or mature or maturing oligodendrocytes $\left(\mathrm{OLIG}^{+}\right.$; PDGFRA $\left.{ }^{-}\right)(\mathbf{B})$ in the cerebellar white matter of control (CON, white shaded bars) or CSF1 OE (OE, grey shaded bars) at p14 and 1 year. RT-qPCR for Pdgfra (C), Olig2 (D), and Mog (E) on mRNA isolated from half brain hemispheres of control (CON, white shaded bars) or CSF1 OE (OE, grey shaded bars) mice at p14 and 1 year. ns = non-significant ( $p$ > 0.05), unpaired, two-tailed $t$ test

quantified in the cerebellar white matter of CSF1 OE and control mice. This region was chosen because $\mathrm{OLIG}^{+}$cells were robustly depleted there in neonatal mice treated with a CSF1R inhibitor [9], and increased numbers of microglia were also observed in this brain region in both p14 and 1-year CSF1 OE mice compared to controls (Supplemental Figure 5). There were no differences in $\mathrm{OLIG}_{2}^{+}$; $\mathrm{PDGFRA}^{+}$or $\mathrm{OLIG}^{+}$; $\mathrm{PDGFRA}^{-}$ cells in CSF1 OE mice compared to controls at either age (Fig. 4A, B; representative images in Supplemental Figure 6), and the proliferation rates of these cell types were also not statistically different between groups (Supplemental Figure 7). Similar results were found in the cortex (Supplemental Figure 8). Furthermore, RTqPCR for markers of both oligodendrocyte precursor cells (Pdgfra and Olig2) as well as mature oligodendrocytes $(M o g)$ did not detect differences between CSF1 OE and control mice (Fig. 4C) at either p14 or 1 year. Therefore, CSF1 OE and the resulting increase in $\mathrm{IBA}^{+}$cells do not appear to impact oligodendrocyte lineage cells.

\section{CSF1 OE does not impact the expression of astrocyte markers}

Some studies of CSF1R inhibitors have observed increased expression of astrocytic markers such as Gfap in response to the drug [8], and activated microglia have been shown to induce the formation of "A1"-activated astrocytes [31]. To determine if increasing CSF1 levels and microglia would also impact $\mathrm{GFAP}^{+}$astrocyte numbers, GFAP ${ }^{+}$cells were also quantified in the cerebellar white matter of CSF1 OE and control mice. No differences were observed in the percentage of cells that are $\mathrm{GFAP}^{+}$in between the two groups at both p14 and 1 year (Fig. 5A; representative images in Supplemental Figure 9). Proliferating $\left(\mathrm{Ki}^{+} 7^{+}\right) \mathrm{GFAP}^{+}$astrocytes were very rare in p14 mice and not detected in 1-year-old mice (data not shown). Furthermore, no differences in expression of Gfap or Slc1a2 (also known as Glt1, a glutamate transporter with enriched expression in astrocytes) were detected by RT-qPCR between CSF1 OE and control mice at either p14 or 1 year (Fig. 5B, C). Additionally, CSF1 OE did not impact expression levels of the "A1" astrocyte marker C3 at either p14 or 1 year of age (Fig. 5D). Therefore, CSF1 OE and the resulting increase in
$\mathrm{IBA}^{+}$cells do not appear to promote $\mathrm{GFAP}^{+}$astrocyte expansion or activation.

\section{Discussion}

Normal adult microglial numbers have been found to be maintained by equivalent apoptotic and proliferative rates. Blocking apoptosis does increase microglial numbers, but numbers eventually stabilize [7]. Our observation that $\mathrm{IBA}^{+}$cell proliferation rates decline over time in CSF1 OE mice indicates that a similar phenomenon occurs in the presence of a pro-proliferative stimulus. It is possible that there are mechanisms in place by which the brain is capable of sensing and responding to abnormal microglial density. It is also possible that CSF1induced proliferation eventually leads to microglial senescence [32], or that older microglia respond differently to the CSF1 mitogenic signal. Additional studies will be required to distinguish between these possibilities.

In a murine glioma model where Csf1 expression is increased approximately 2.5-fold compared to normal brain, CSF1R inhibitors decrease expression of M2 markers including Arg1 in GAMs [17, 18], suggesting that increased CSF1/CSF1R signaling can promote polarization toward a M2 phenotype in the diseased CNS. However, in CSF1 OE mice we do not find evidence for increased expression of the commonly used M2 polarization markers ARG1 and MRC1 (CD206), even in aged mice. Gliomas produce other factors that signal to macrophage lineage cells, so one possible explanation is that increased levels of CSF1 alone are unable to increase expression of M2 polarization genes but can do so when combined with other signals.

RNA-seq data indicates that increased CSF1 signaling influences transcription of a relatively limited number of genes in microglia, several of which are related to protein synthesis. In bone marrow-derived macrophages in vitro, CSF1 has been shown to promote protein synthesis [12] and proliferating cells require increased protein synthesis. Additionally, "cellular response to interferon gamma (IFN- $\gamma$ )" is one of the GO terms enriched in microglia from CSF1 OE mice. Given that IFN- $\gamma$ is one of the stimuli used to polarize to a M1 phenotype [13], CSF1 OE microglia could therefore be considered to have some M1 characteristics. However, RNA-seq data indicate that the commonly used M1 marker Nos2 (iNOS) is not differentially expressed in 
a

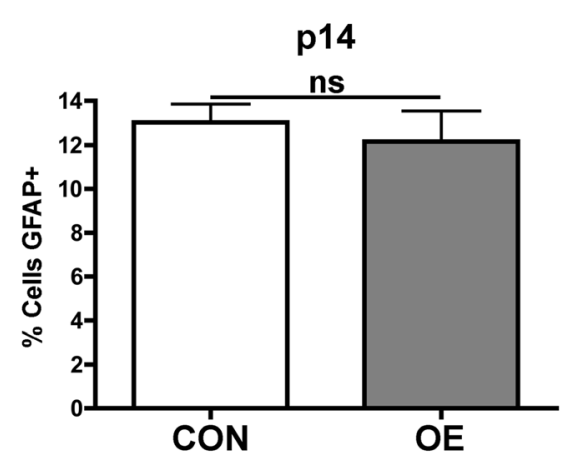

b

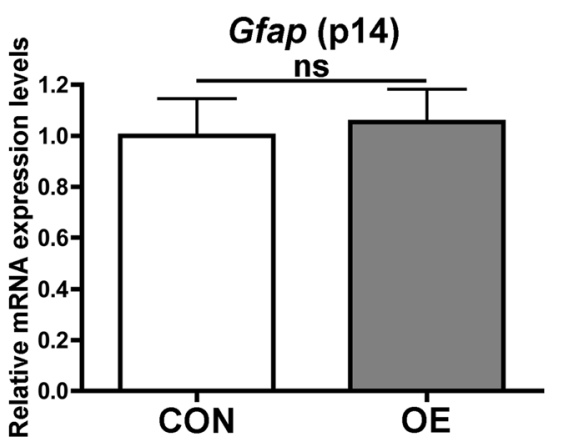

C

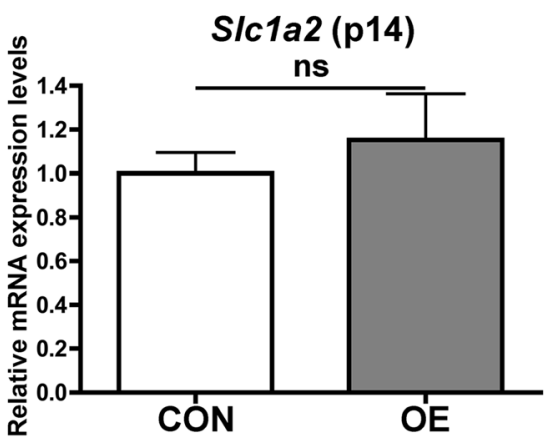

d

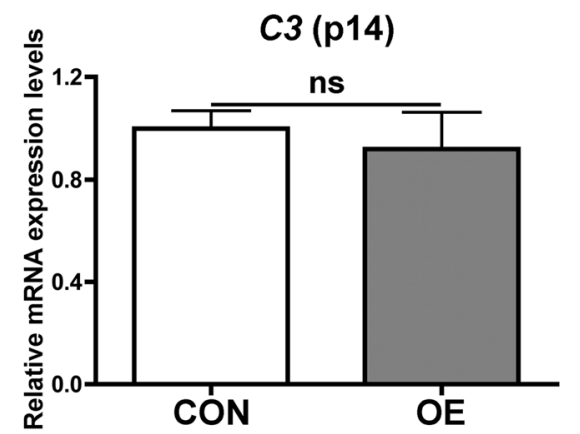

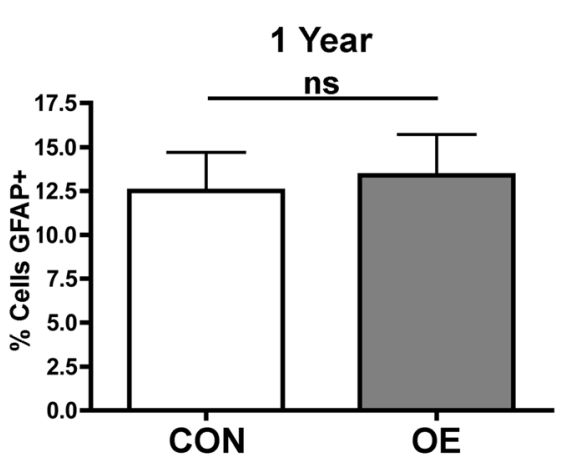
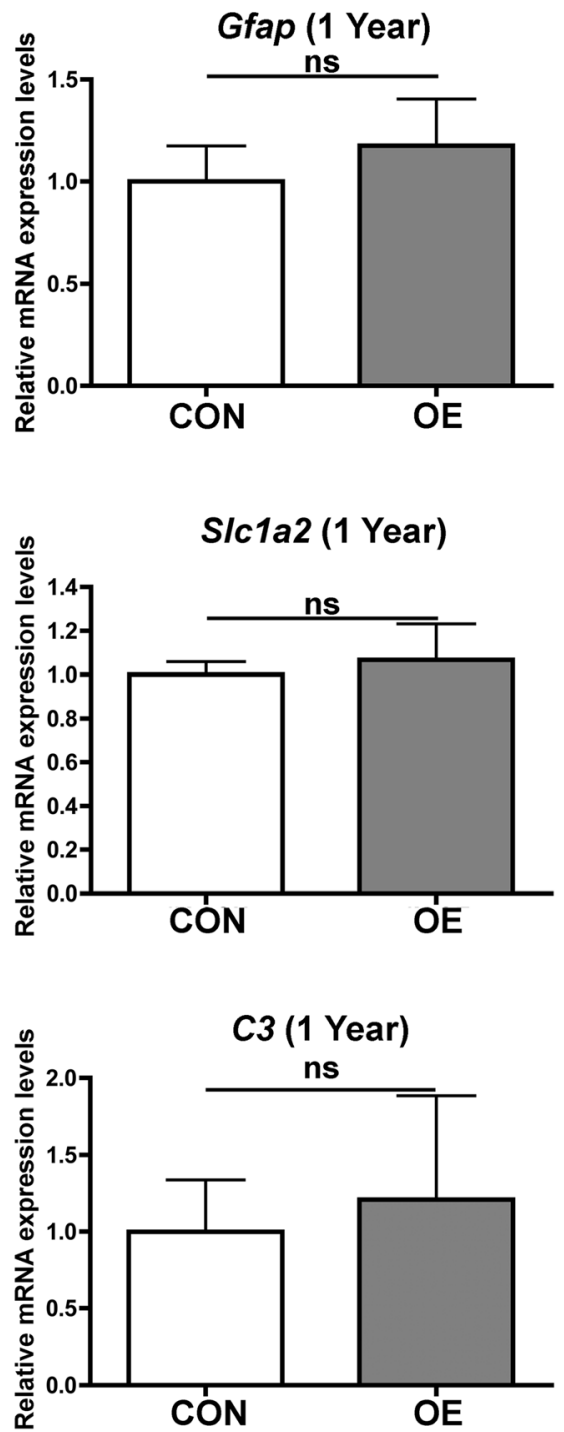

Fig. 5 CSF1 OE does not impact the expression of astrocytic genes. A Quantification of the percent of cells that are GFAP ${ }^{+}$astrocytes in the cerebellar white matter of control (CON, white shaded bars) or CSF1 OE (OE, grey shaded bars) at p14 and 1 year. RT-qPCR for Gfap (B), SIC1a2 (C), and C3 (D) on mRNA isolated from half brain hemispheres of control (CON, white shaded bars) or CSF1 OE (OE, grey shaded bars) mice at age p14 or 1 year. ns = non-significant $(p>0.05)$, unpaired, two-tailed $t$ test 
CSF1 OE microglia compared to control. Therefore, our data indicate that in vivo, CSF1 OE promotes a gene expression state in microglia that falls on the continuum between M1 and M2. Moreover, it is possible that CSF1 signaling is responsible for some of the previously recognized expression of interferon targets that occurs in microglia in normal mice [33]. Further studies will be necessary to fully elucidate how CSF1 OE influences immune responses in the CNS.

Microglial actions are known to have impacts on the oligodendrocyte lineage. For example, microglial specific deletion of transglutaminase 2 decreased OPC proliferation and caused a reduction in OPC and oligodendrocyte numbers in otherwise wild-type mice [23]. Our studies indicate that increasing $\mathrm{IBA} 1^{+}$cells does not have the converse effect. It is possible that in the normal brain, microglial actions supporting oligodendrogenesis are already "saturated" and that further increasing their number has no impact. Alternatively, CSF1 OE may produce a state in $\mathrm{IBA}^{+}$cells that renders them incapable of providing oligodendrocyte support.

Similarly, in CNS injury or disease, both increased microglial numbers and an astroglial reaction are commonly observed. We utilized the marker GFAP to examine if CSF1 OE impacts astrocyte numbers. One limitation to our study is that some astrocytes, particularly those in the grey matter, do not express levels of GFAP that are detected by immunohistochemistry [34] and therefore would not have been detected by our methods. Upon activation, astrocytes can take on different phenotypes, and one such phenotype termed "A1" is induced by interleukin $1 \alpha$ (Il-1 $1 \alpha)$; tumor necrosis factor (TNF); and complement component 1 , subcomponent q (C1q) produced by activated microglia [31]. Our RNAseq data did not find increased expression of transcripts encoding these factors in microglia and by RT-qPCR, we did not observe increased expression of the "A1" marker C3. Our data, therefore, support the hypothesis that altered microglial function, and not simply increased microglial density, contributes to the astrogliosis that occurs in CNS pathologies.

\section{Conclusions}

In summary, our studies found no impact of CSF1 overexpression alone on glia outside of microglia. However, in situations of CNS disease or injury where multiple inflammatory mediators are produced, CSF1 overexpression could act together with other factors to have additional impacts.

\section{Abbreviations}

ARG1: Arginase 1; C3: Complement C3; CON: Control; CNS: Central nervous system; CSF1: Colony-stimulating factor 1; CSF1R: Colony-stimulating factor 1 receptor; DE: Differentially expressed; FDR: False discovery rate; FC: Fold change; GAM: Glioma-associated macrophages/microglia; IBA1: Ionized calcium-binding adaptor molecule 1; IFN-ץ: Interferon gamma; IL34: Interleukin -34; MRC1: Mannose receptor C-type 1; Ns: Not significant; ND: Not detected; OE: Overexpressing; OLIG2: Oligodendrocyte transcription factor 2; PDGFRA: Platelet-derived growth factor receptor A; PPDE: Posterior probability of differential expression; RT-qPCR: Reverse transcription, qualitative PCR; Seq: Sequencing; TNF: Tumor necrosis factor

\section{Supplementary Information}

The online version contains supplementary material available at https://doi. org/10.1186/s12974-021-02212-0.

Additional file 1: Supplemental Figure 1. Representative images for IBA1 and Ki67 immunofluorescence. Genotype, age, and brain region are indicated for each image. Scale bar $=50$ microns.

Additional file 2: Supplemental Figure 2. Transgene expression in CSF1 OE mice. RT-qPCR indicates increased Csf1 levels in CSF1 OE (grey bars) mice compared to control mice (white bars) at both p14 (A) and 1 year (B). No-RT reactions were included for all samples and no amplification was detected (not shown). $\mathrm{N}=3-5$ mice per group. ${ }^{* *}=p<0.001$; ${ }^{* * * *}=p<0.0001$; unpaired, two-tailed t-test. (C) Representative images showing expression of EGFP (green) in 1 year CSF1 OE mice. Scale bar = 50 microns. (D) Representative images showing detection of CSF1 protein by immunofluorescence in a subset of $\mathrm{GFAP}^{+}$cells in 1-year old CSF1 OE mice but not control mice. Asterisks= examples of autofluorescence of red blood cells; arrows $=$ examples of $\mathrm{CSF}^{+}{ }^{+} \mathrm{GFAP}^{+}$cells. Scale bar $=20$ microns.

Additional file 3: Supplemental Figure 3. Representative images for IBA1 and TUNEL immunofluorescence. Genotype, age, and brain region are indicated for each image. Scale bar $=50$ microns

Additional file 4: Supplemental Figure 4. ARG1 antibody validation ARG1 (green) and IBA1 (red) immunofluorescence staining in a murine glioma. Arrow indicates an example ARG $1^{+} \mid \mathrm{BA} 1^{+}$cell. Scale bar $=25$ microns.

Additional file 5: Supplemental Figure 5. $\mid \mathrm{BA} 1^{+}$cell numbers are increased in the cerebellar white matter of CSF1 OE mice. Quantification of the percent of cells that are $\mathrm{IBA}^{+}$in (CON, white bars) and CSF1 OE $\left(\mathrm{OE}\right.$, grey bars) mice at p14 (A) and 1 year $(\mathrm{B}) .{ }^{* *}=p<0.01$

Additional file 6: Supplemental Figure 6. Representative images for OLIG2, PDGFRA, and Ki67 immunofluorescence. Genotype and age are indicated for each image while dots indicate the edge of cerebellar white matter. Scale bar $=50$ microns

Additional file 7: Supplemental Figure 7. Proliferation rates of oligodendrocyte lineage cells in the cerebellar white matter do not differ between control (CON, white bars) and CSF1 OE (OE, grey bars) mice. Quantification of the percent of OPCS (PDGFRA ${ }^{+} ; \mathrm{OLIG}^{+}$) (A) or mature or maturing oligodendrocytes (OLIG2+ ${ }^{+}$PDGFRA') (B) cells that are proliferating $\left(\mathrm{Ki}_{6} \mathrm{7}^{+}\right)$at $\mathrm{p} 14$. No proliferating oligodendrocyte lineage cells were observed in 1-year old mice of either genotype. ns= non-significant ( $p>0.05)$, unpaired, two-tailed t-test.

Additional file 8: Supplemental Figure 8. $\mid \mathrm{BA} 1^{+}$cells are increased but there are no differences in oligodendrocyte lineage cells in the cortex of CSF1 OE mice. Quantification of the percent of cells that are $\mid \mathrm{BA} 1^{+}(\mathrm{A}), \mathrm{OLIG} 2^{+} ; \mathrm{PDGFRA}^{+}(\mathrm{OPCS})(\mathrm{B})$, and mature or maturing oligodendrocytes (OLIG2+ ${ }^{+}$PDGFRA') (C) cells in control (CON, white shaded bars), and CSF1 OE (OE, grey shaded bars) mice at p14 and 1 year. D) Quantification of the percent of OPCs (PDGFRA ${ }^{+}$; OLIG2 ${ }^{+}$) or mature or maturing oligodendrocytes (OLIG2+ ${ }^{+}$PDGFRA $\left.{ }^{-}\right)$cells that are proliferating $\left(\mathrm{Ki}_{6} \mathrm{7}^{+}\right)$in control (CON, white shaded bars) and CSF1 OE (OE, grey shaded bars) mice at p14. No proliferating oligodendrocyte lineage cells were observed in 1-year old mice of either genotype. ns= non-significant $(p>0.05),{ }^{*}=p<0.05,{ }^{* *}=p<0.01$, unpaired, two-tailed t-test.

Additional file 9: Supplemental Figure 9. Representative images for GFAP and Ki67 immunofluorescence. Genotype and age are indicated for each image while dots indicate the edge of cerebellar white matter. Scale bar $=50$ microns. 
Additional file 10: Supplemental Table 1. Genes that were found to be differentially expressed in microglia from CSF1 OE mice compared to control (CON). PPDE= posterior probability of differential expression, $\mathrm{FC}=$ fold change. Normalized expected counts are shown for each gene for each of four samples from the two genotypes.

\section{Acknowledgements}

We thank the Johnson, Taylor, Bashirullah, and Marker laboratories for the use of equipment. We thank the Watters laboratory for sharing protocols. The authors thank the University of Wisconsin Biotechnology Center Gene Expression Center for providing Illumina RNA library preparation and the DNA Sequencing Facility for sequencing services.

\section{Authors' contributions}

I.D., M.B, C.K., and L.C. were involved in study design. M.B., S.L., V.M., and L.C performed immunohistochemical experiments and imaging. I.D., S.L., and V.M. performed animal work. L.K. and V.M. performed mRNA work. Z.W., C.K. and L.C. analyzed data. M.B., C.K., and L.C. wrote the manuscript. All authors have reviewed and approved of the manuscript.

\section{Funding}

This research was supported by the University of Wisconsin Carbone Cancer Center Support Grant P30 CA014520 (Collier and Kendziorski), R01 NS085364 (Collier), the Molecular and Cellular Pharmacology training grant T32 GM008688 (Boyd), and by GM102756 (Kendziorski). The funding bodies had no role in the design of the study or in the collection, analysis, and interpretation of the data.

\section{Availability of data and materials}

RNA-sequencing data has been deposited at GEO (accession number GSE151698). Other data from this manuscript is available from the corresponding author upon reasonable request.

\section{Declarations}

\section{Ethics approval and consent to participate}

Animal experiments were conducted in accordance with the United States National Research Council's Guide for the Care and Use of Laboratory Animals and under the approval of the Institutional Animal Care and Use Committee at the University of Wisconsin-Madison.

\section{Consent for publication}

Not applicable.

\section{Competing interests}

The authors declare that they have no competing interests.

\section{Author details}

'Molecular and Cellular Pharmacology Graduate Program, University of Wisconsin, Madison, USA. ${ }^{2}$ Pharmaceutical Sciences Division, School of Pharmacy, University of Wisconsin, Madison, USA. ${ }^{3}$ Department of Statistics, University of Wisconsin, Madison, USA. ${ }^{4}$ Department of BiostatisticsUniversity of Wisconsin, Madison, USA. ${ }^{5}$ University of Wisconsin Carbone Comprehensive Cancer Center, Madison, USA.

\section{Received: 19 January 2021 Accepted: 5 July 2021}

Published online: 19 July 2021

\section{References}

1. Erblich B, Zhu L, Etgen AM, Dobrenis K, Pollard JW. Absence of colony stimulation factor-1 receptor results in loss of microglia, disrupted brain development and olfactory deficits. PLoS ONE. 2011;6(10):e26317. https:// doi.org/10.1371/journal.pone.0026317.

2. Wang Y, Szretter KJ, Vermi W, Gilfillan S, Rossini C, Cella M, et al. IL-34 is a tissue-restricted ligand of CSF1R required for the development of Langerhans cells and microglia. Nat Immunol. 2012;13(8):753-60. https://doi. org/10.1038/ni.2360.

3. Nandi S, Gokhan S, Dai XM, Wei S, Enikolopov G, Lin H, et al. The CSF-1 receptor ligands IL-34 and CSF-1 exhibit distinct developmental brain expression patterns and regulate neural progenitor cell maintenance and maturation. Dev Biol. 2012;367(2):100-13. https://doi.org/10.1016/j.ydbio.2 012.03.026.

4. Kana V, Desland FA, Casanova-Acebes M, Ayata P, Badimon A, Nabel E, et al. CSF-1 controls cerebellar microglia and is required for motor function and social interaction. J Exp Med. 2019;216(10):2265-81. https://doi.org/10.1084/ jem.20182037.

5. Easley-Neal C, Foreman O, Sharma N, Zarrin AA, Weimer RM. CSF1R ligands IL-34 and CSF1 are differentially required for microglia development and maintenance in white and gray matter brain regions. Front Immunol. 2019; 10:2199. https://doi.org/10.3389/fimmu.2019.02199.

6. Nikodemova M, Kimyon RS, De I, Small AL, Collier LS, Watters JJ. Microglial numbers attain adult levels after undergoing a rapid decrease in cell number in the third postnatal week. J Neuroimmunol. 2015;278:280-8. https://doi.org/10.1016/j.jneuroim.2014.11.018.

7. Askew K, Li K, Olmos-Alonso A, Garcia-Moreno F, Liang Y, Richardson P, et al. Coupled proliferation and apoptosis maintain the rapid turnover of microglia in the adult brain. Cell Rep. 2017;18(2):391-405. https://doi.org/1 0.1016/j.celrep.2016.12.041.

8. Elmore MR, Najafi AR, Koike MA, Dagher NN, Spangenberg EE, Rice RA, et al. Colony-stimulating factor 1 receptor signaling is necessary for microglia viability, unmasking a microglia progenitor cell in the adult brain. Neuron. 2014;82(2):380-97. https://doi.org/10.1016/j.neuron.2014.02.040.

9. Hagemeyer N, Hanft KM, Akriditou MA, Unger N, Park ES, Stanley ER, et al. Microglia contribute to normal myelinogenesis and to oligodendrocyte progenitor maintenance during adulthood. Acta Neuropathol. 2017;134(3): 441-58. https://doi.org/10.1007/s00401-017-1747-1.

10. De I, Steffen MD, Clark PA, Patros CJ, Sokn E, Bishop SM, et al. CSF1 overexpression promotes high-grade glioma formation without impacting the polarization status of glioma-associated microglia and macrophages. Cancer Res. 2016;76(9):2552-60. https:/doi.org/10.1158/0008-5472.CAN-15-2386.

11. Du Yan S, Zhu H, Fu J, Yan SF, Roher A, Tourtellotte WW, et al. Amyloid-beta peptide-receptor for advanced glycation endproduct interaction elicits neuronal expression of macrophage-colony stimulating factor: a proinflammatory pathway in Alzheimer disease. Proc Natl Acad Sci U S A. 1997;94(10):5296-301. https://doi.org/10.1073/pnas.94.10.5296.

12. Luo J, Elwood F, Britschgi M, Villeda S, Zhang H, Ding Z, et al. Colonystimulating factor 1 receptor (CSF1R) signaling in injured neurons facilitates protection and survival. J Exp Med. 2013;210(1):157-72. https://doi.org/10.1 084/jem.20120412.

13. Sica A, Mantovani A. Macrophage plasticity and polarization: in vivo veritas. J Clin Invest. 2012;122(3):787-95. https://doi.org/10.1172/JC159643.

14. Martinez FO, Gordon S, Locati M, Mantovani A. Transcriptional profiling of the human monocyte-to-macrophage differentiation and polarization: new molecules and patterns of gene expression. J Immunol. 2006;177(10):730311. https://doi.org/10.4049/jimmunol.177.10.7303.

15. Verreck FA, de Boer T, Langenberg DM, Hoeve MA, Kramer M, Vaisberg E, et al. Human IL-23-producing type 1 macrophages promote but IL-10-producing type 2 macrophages subvert immunity to (myco)bacteria. Proc Natl Acad Sci U S A. 2004;101(13):4560-5. https://doi.org/10.1073/pnas.0400983101.

16. Fleetwood AJ, Lawrence T, Hamilton JA, Cook AD. Granulocyte-macrophage colony-stimulating factor (CSF) and macrophage CSF-dependent macrophage phenotypes display differences in cytokine profiles and transcription factor activities: implications for CSF blockade in inflammation. J Immunol. 2007: 178(8):5245-52. https://doi.org/10.4049/jimmunol.178.8.5245.

17. Yan D, Kowal J, Akkari L, Schuhmacher AJ, Huse JT, West BL, et al. Inhibition of colony stimulating factor-1 receptor abrogates microenvironmentmediated therapeutic resistance in gliomas. Oncogene. 2017;36(43):6049-58. https://doi.org/10.1038/onc.2017.261.

18. Pyonteck SM, Akkari L, Schuhmacher AJ, Bowman RL, Sevenich L, Quail DF, et al. CSF-1R inhibition alters macrophage polarization and blocks glioma progression. Nat Med. 2013;19(10):1264-72. https://doi.org/10.1038/nm.3337.

19. Xue J, Schmidt SV, Sander J, Draffehn A, Krebs W, Quester I, et al. Transcriptome-based network analysis reveals a spectrum model of human macrophage activation. Immunity. 2014;40(2):274-88. https://doi.org/10.101 6/j.immuni.2014.01.006.

20. Nahrendorf M, Swirski FK. Abandoning M1/M2 for a Network Model of Macrophage Function. Circ Res. 2016;119(3):414-7. https://doi.org/10.1161/ CIRCRESAHA. 116.309194

21. De I, Nikodemova M, Steffen MD, Sokn E, Maklakova VI, Watters JJ, et al. CSF1 overexpression has pleiotropic effects on microglia in vivo. Glia. 2014; 62(12):1955-67. https://doi.org/10.1002/glia.22717. 
22. Liu Y, Given KS, Dickson EL, Owens GP, Macklin WB, Bennett JL. Concentration-dependent effects of CSF1R inhibitors on oligodendrocyte progenitor cells ex vivo and in vivo. Exp Neurol. 2019;318:32-41. https://doi. org/10.1016/j.expneurol.2019.04.011

23. Giera S, Luo R, Ying Y, Ackerman SD, Jeong SJ, Stoveken HM, et al. Microglial transglutaminase-2 drives myelination and myelin repair via GPR56/ADGRG1 in oligodendrocyte precursor cells. Elife. 2018;7:e33385. https://doi.org/10.7554/eLife.33385.

24. Nicholas RS, Wing MG, Compston A. Nonactivated microglia promote oligodendrocyte precursor survival and maturation through the transcription factor NF-kappa B. Eur J Neurosci. 2001;13(5):959-67. https:// doi.org/10.1046/j.0953-816x.2001.01470.X.

25. Schindelin J, Arganda-Carreras I, Frise E, Kaynig V, Longair M, Pietzsch T, et al. Fiji: an open-source platform for biological-image analysis. Nat Methods. 2012;9(7):676-82. https://doi.org/10.1038/nmeth.2019.

26. Schneider CA, Rasband WS, Eliceiri KW. NIH Image to ImageJ: 25 years of image analysis. Nat Methods. 2012;9(7):671-5. https://doi.org/10.1038/ nmeth.2089.

27. Huang da W, Sherman BT, Lempicki RA. Systematic and integrative analysis of large gene lists using DAVID bioinformatics resources. Nat Protoc. 2009; 4(1):44-57. https://doi.org/10.1038/nprot.2008.211.

28. Huang da W, Sherman BT, Lempicki RA. Bioinformatics enrichment tools: paths toward the comprehensive functional analysis of large gene lists. Nucleic Acids Res. 2009:37(1):1-13. https://doi.org/10.1093/nar/gkn923.

29. Sharma N, Akkoyunlu M, Rabin RL. Macrophages-common culprit in obesity and asthma. Allergy. 2018;73(6):1196-205. https://doi.org/10.1111/all.13369.

30. Murray PJ. Macrophage Polarization. Annu Rev Physiol. 2017;79(1):541-66. https://doi.org/10.1146/annurev-physiol-022516-034339.

31. Liddelow SA, Guttenplan KA, Clarke LE, Bennett FC, Bohlen CJ, Schirmer L, et al. Neurotoxic reactive astrocytes are induced by activated microglia. Nature. 2017;541(7638):481-7. https://doi.org/10.1038/nature21029.

32. Hu Y, Fryatt GL, Ghorbani M, Obst J, Menassa DA, Martin-Estebane M, et al. Replicative senescence dictates the emergence of disease-associated microglia and contributes to A pathology. Cell Rep. 2021;35(10):109228. https://doi.org/10.1016/j.celrep.2021.109228.

33. Goldmann T, Zeller N, Raasch J, Kierdorf K, Frenzel K, Ketscher L, et al. USP18 lack in microglia causes destructive interferonopathy of the mouse brain. EMBO J. 2015;34(12):1612-29. https://doi.org/10.15252/embj.201490791.

34. Khakh BS, Sofroniew MV. Diversity of astrocyte functions and phenotypes in neural circuits. Nat Neurosci. 2015;18(7):942-52. https://doi.org/10.1038/nn.4043.

\section{Publisher's Note}

Springer Nature remains neutral with regard to jurisdictional claims in published maps and institutional affiliations.

Ready to submit your research? Choose BMC and benefit from:

- fast, convenient online submission

- thorough peer review by experienced researchers in your field

- rapid publication on acceptance

- support for research data, including large and complex data types

- gold Open Access which fosters wider collaboration and increased citations

- maximum visibility for your research: over $100 \mathrm{M}$ website views per year

At $\mathrm{BMC}$, research is always in progress.

Learn more biomedcentral.com/submissions 